\title{
Finite codimensional maximal ideals in subalgebras of ultrametric uniformly continuous functions
}

\author{
by Monique Chicourrat, Bertin Diarra and Alain Escassut
}

\begin{abstract}
Let $\mathbb{E}$ be a complete ultrametric space, let $\mathbb{K}$ be a perfect complete ultrametric field and let $A$ be a Banach $\mathbb{K}$-algebra which is either a full $\mathbb{K}$-subalgebra of the algebra of continuous functions from $\mathbb{E}$ to $\mathbb{K}$ owning all characteristic functions of clopens of $\mathbb{E}$, or a full $\mathbb{K}$-subalgebra of the algebra of uniformly continuous functions from $\mathbb{E}$ to $\mathbb{K}$ owning all characteristic functions of uniformly open subsets of $\mathbb{E}$. We prove that all maximal ideals of finite codimension of $A$ are of codimension 1.
\end{abstract}

Introduction: Let $\mathbb{E}$ be a complete metric space provided with an ultrametric distance $\delta$, let $\mathbb{K}$ be a perfect complete ultrametric field and let $S$ be a full $\mathbb{K}$-subalgebra of the $\mathbb{K}$-algebra of continuous (resp. uniformly continuous) functions complete with respect to an ultrametric norm $\|$. $\|$ that makes it a Banach $\mathbb{K}$-algebra [3].

In [2], [4], [5], [6] we studied several examples of Banach IK-algebras of functions and showed that for each example, each maximal ideal is defined by ultrafilters [1], [7], [8] and that each maximal ideal of finite codimension is of codimension 1: that holds for continuous functions [4] and for all examples of functions we examine in [2], [5], [6]. Thus, we can ask whether this comes from a more general property of Banach $\mathbb{K}$-algebras of functions, what we will prove here.

Here we must assume that the ground field $\mathbb{K}$ is perfect, which makes that hypothesis necessary in all theorems.

Definitions and notations: A subset $D$ of $\mathbb{E}$ is said to be clopen (resp.uniformly open) if it is closed and open (resp. if $\inf _{x \in D} \delta(x, y)>0$ ).

The algebra $S$ will be said to be semi-admissiible if it satisfies the two following properties:

1) For every clopen subset $D$ of $\mathbb{E}$, the characteristic function of $D$ belongs to $S$,

2) For every $f \in S$ such that $\inf _{\mathbb{E}}|f(x)|>0, f$ is invertible in $S$.

The algebra $S$ will be said to be semi-compatible [2] if it satisfies the two following properties: to $S$,

1) For every uniformly open subset $D$ of $\mathbb{E}$, the characteristic function of $D$ belongs

2) For every $f \in A$ such that $\inf _{\mathbb{E}}|f(x)|>0, f$ is invertible in $S$.

Examples: 1) The Banach algebra of all bounded continuous functions from $\mathbb{E}$ to $\mathbb{K}$, provided with the uniform norm $\|$. $\|_{0}$ on $\mathbb{E}$, is semi-admissible.

2) Let $A$ be the algebra of bounded functions from $\mathbb{E}$ to $\mathbb{K}$ such that, for every $a \in \mathbb{E}$, there exists a ball $B(a)=\{x \in \mathbb{E} \mid \delta(a, x)<r(a)\}$ such that $f(x)=f(a) \forall x \in B(a)$. 
Then the closure of $A$ with respect to the uniform norm $\|$. $\|_{0}$ is easily seen to be a semi-admissible algebra (different from Example 1) whenever $\mathbb{E}$ is not separable).

3) Let $B$ be the Banach algebra of bounded uniformly continuous functions from $\mathbb{E}$ to $\mathbb{I K}$ provided with the uniform norm $\|.\|_{0}$ on $\mathbb{E}$. Then $B$ is a semi-compatible algebra.

4) Let $L$ be the algebra of bounded Lipschitzian functions from $\mathbb{E}$ to $\mathbb{K}$ provided with the norm $\|\cdot\|$ defined as $\|f\|=\max \left(\|f\|_{0}, \sup _{x, y \in \mathbb{E}, x \neq y} \frac{|f(x)-f(y)|}{\delta(x, y)}\right)$. Then $L$ is proved to be a semi-compatible $\mathbb{I K}$-Banach algebra [2].

Throughout the paper, we denote by $S$ a $\mathbb{K}$-algebra that is either semiadmissible or semi-compatible.

The following Theorem A plays a major role in the proof of our theorems. Concerning semi-compatible algebras, it just comes from Theorem 3.1 in [2]. Concerning semi-adimissible algebras, we can generalize Theorem 5.1 in [4] and then Corollary 5.2 to any semi-admissible algebras. Thus, we obtain Theorem A:

Theorem A: Let $\mathcal{M}$ be a maximal ideal of $S$. There exist ultrafilters $\mathcal{U}$ on $\mathbb{E}$ such that $\mathcal{M}$ is the set of $f \in S$ such that $\lim _{\mathcal{U}} f(x)=0$. Moreover, if every $f \in S$ converges along $\mathcal{U}$, then $\mathcal{M}$ is of codimension 1 .

In order to prove our main theorems we first need several basic results. In [5], [6], we have proved a proposition that we can easily generalize:

Proposition P1: $\quad$ Let $\mathbb{L}$ be a finite algebraic extension of $\mathbb{K}$ provided with the absolute value which extends that of $\mathbb{K K}$. Let $A$ be a unital commutative $\mathbb{K}$-algebra. Suppose there exists a morphism of $\mathbb{K}$-algebra, $\chi$, from $A$ onto $\mathbb{L}$. Let $\widehat{A}$ be the $\mathbb{L}$-algebra $\mathbb{L} \otimes_{\mathbb{K}} A$. Then $\chi$ has continuation to a surjective morphism of $\mathbb{L}$-algebra $\widehat{\chi}$ from $\widehat{A}$ to $\mathbb{L}$.

Proof: The above theorem is given as Theorem 3.5 in [5], but on the one hand, the proof was made by using the primitive element theorem, though the field is not supposed to be perfect (but one can generalize it when the field is not perfect). On the other hand the surjective property was not specified.

Let $d=[\mathbb{L}: \mathbb{K}]$. Since $\mathbb{K}$ is perfect we can suppose that $\mathbb{L}$ is of the form $\mathbb{K}[a]$. Let $f, g \in \widehat{A}$. Then $f$ is of the form $\sum_{j=0}^{d-1} a^{j} f_{j}, f_{j} \in S, j=0, \ldots, d-1$ and $g$ is of the form

$$
\sum_{j=0}^{d-1} a^{j} g_{j}, g_{j} \in S, j=0, \ldots, d-1
$$

We can now define $\widehat{\chi}$ on $\widehat{A}$ as $\widehat{\chi}(f)=\sum_{j=0}^{d-1} a^{j} \chi\left(f_{j}\right)$. Then obviously, $\widehat{\chi}$ is IK-linear. On the other hand, for each $q \in \mathbb{N}, a^{q}$ is of the form $P_{q}(a)$ where $P_{q} \in \mathbb{K}[x], \operatorname{deg}\left(P_{q}\right) \leq d-1$. 
Then $\widehat{\chi}\left(a^{q}\right)=\widehat{\chi}\left(P_{q}(a)\right)=P_{q}(\widehat{\chi}(a))=P_{q}(a)=a^{q}$. Next,

$$
\begin{gathered}
\widehat{\chi}(f g)=\widehat{\chi}\left(\left(\sum_{j=0}^{d-1} a^{j} f_{j}\right)\left(\sum_{j=0}^{d-1} a^{j} g_{j}\right)\right)=\widehat{\chi}\left(\sum_{\substack{0 \leq m \leq d-1 \\
0 \leq n \leq d-1}} a^{m+n} f_{m} g_{n}\right) \\
=\sum_{\substack{0 \leq m \leq d-1 \\
0 \leq n \leq d-1}} a^{m+n} \chi\left(f_{m}\right) \chi\left(g_{n}\right)=\left(\sum_{j=0}^{d-1} a^{j} \chi\left(f_{j}\right)\right)\left(\sum_{j=0}^{d-1} a^{j} \chi\left(g_{j}\right)\right)=\chi(f) \chi(g) .
\end{gathered}
$$

Thus, the extension of $\chi$ is proved. It is then immediately checked that $\widehat{\chi}$ is surjective: since $\widehat{A}$ is a $\mathbb{L}$-algebra, it contains the field $\mathbb{L}$ and every morphism $\widehat{\chi}$ from $\widehat{A}$ obviously satisfies $\widehat{\chi}(c)=c \forall c \in \mathbb{L}$.

Remark: Proposition P1 holds in under much more general hypotheses, without assuming that $\mathbb{K}$ is perfect and that $\mathbb{L}$ is algebraic over $\mathbb{K}$.

The following proposition P2 is essential for establishing P3 and all theorems:

Proposition P2: $\quad$ Let $\mathbb{L}=\mathbb{K}[a]$ be a finite extension of $\mathbb{K}$ of degree $t$, provided with the unique absolute value extending that of $\mathbb{K}$ and let $a_{2}, \ldots, a_{t}$ be the conjugates of a over $\mathbb{K}$, with $a_{1}=a$. Let $\widehat{S}=\mathbb{L} \otimes_{\mathbb{K}} S$ and let $g=\sum_{j=0}^{t-1} a^{j} f_{j}, f_{j} \in S$ be such that $\inf _{\mathbb{E}}|g(x)|>0$. For every $k=1, \ldots, t$, let $g_{k}=\sum_{j=0}^{t-1} a_{k}^{j} f_{j}, f_{j} \in S$. Then $\prod_{k=1}^{t} g_{k}$ belongs to $S$ and $\prod_{k=2}^{t} g_{k}$ belongs to $\widehat{S}$.

Proof: Since $\mathbb{L}=\mathbb{K}\left[a_{1}\right]$ by definition, we have

$$
N=\mathbb{K}\left[a_{1}, \ldots \ldots ., a_{t}\right]=\mathbb{K}\left[a_{1}\right]\left[a_{2}, \ldots \ldots . ., a_{t}\right]=\mathbb{L}\left[a_{2}, \ldots \ldots \ldots, a_{t}\right]
$$

and $N$ is a normal extension of $\mathbb{K}$ and $\mathbb{L}$ respectively.

Thus, assuming that $a_{1}, \ldots \ldots \ldots, a_{s}$ belong to $\mathbb{L}$, we have $\mathbb{L}=\mathbb{K}\left[a_{1}, \ldots \ldots ., a_{s}\right]$ and $N=\mathbb{K}\left[a_{1} \ldots ., a_{s}, a_{s+1}, \ldots \ldots, a_{t}\right]=\mathbb{K}\left[a_{1}, \ldots \ldots ., a_{s}\right]\left[a_{s+1}, \ldots \ldots ., a_{t}\right]=\mathbb{L}\left[a_{s+1}, \ldots \ldots, a_{t}\right]$.

Put $G^{\prime}=\{\sigma \in G: \sigma(x)=x, \forall x \in L\}$ with the extension $N \mid \mathbb{L}$ which is Galoisian, of Galois group $G^{\prime}=G(N \mid \mathbb{L})$ the subfield $\mathbb{L}$ of $N$ corresponds to the subgroup $G^{\prime}$ of $G$ through the Galois correspondance.

Now, given $\sigma \in G$, set $\sigma(g)=\sum_{j=0}^{t-1}(\sigma(a))^{j} f_{j}$. Let $F=\prod_{k=1}^{t} g_{k}=\prod_{\sigma \in G} \sigma(g)$. Then $F$ belongs to $S$ if and only if for every $\tau \in G, \tau(F)=F$. Now, we have

$$
\tau(F)=\prod_{\sigma \in G} \tau \circ \sigma(g)=\prod_{\zeta \in G} \zeta(g)=F
$$

therefore $F$ belongs to $S$. 
On the other hand the roots $a_{i}$, for $s+1 \leq i \leq t$ are conjugate over $\mathbb{L}$. Therefore if $s+1 \leq i \leq t$, there exists $\theta \in G^{\prime}=G(N \mid \mathbb{L})$ such that $a_{i}=\theta\left(a_{s+1}\right)$. It follows that $g_{i}=\sum_{j=0}^{t-1} a_{i}^{j} f_{j}=\sum_{j=0}^{t-1} \theta\left(a_{s+1}\right)^{j} f_{j}=\theta\left(\sum_{j=0}^{t-1} a_{s+1}^{j} f_{j}\right)=\theta\left(g_{s+1}\right)$

Let $H=\prod_{i=s+1}^{t} g_{i}=\prod_{\theta \in G^{\prime}} \theta\left(g_{s+1}\right)$. Then $H$ belongs to $\widehat{S}$ if and only if $\tau(H)=H \forall \tau \in$ $G^{\prime}$. Now, we have $\tau(H)=\prod_{\theta \in G^{\prime}} \tau \circ \theta\left(g_{s+1}\right)=\prod_{\zeta \in G^{\prime}} \zeta\left(g_{s+1}\right)=H$, therefore $H$ belongs to $\widehat{S}$. Consequently, since $\prod_{i=2}^{s} g_{i}$ belongs to $\widehat{S}$, one gets that $\left(\prod_{i=2}^{s} g_{i}\right) \cdot H=\prod_{i=2}^{t} g_{i}$ is an element of $\widehat{S}$.

We can now establish the following Proposition P3:

Proposition P3: $\quad$ Let $\mathbb{L}=\mathbb{K}[a]$ be a finite extension of $\mathbb{K}$ of degree $t$, provided with the unique absolute value extending that of $\mathbb{K}$ and let $a_{2}, \ldots, a_{t}$ be the conjugates of a over $\mathbb{K}$, with $a_{1}=a$. Let $\widehat{S}=\mathbb{L} \otimes_{\mathbb{K}} S$ and let $g \in \widehat{S}$ be such that $\inf _{\mathbb{E}}|g(x)|>0$. Then $g$ is invertible in $\widehat{S}$.

Proof: Let $g=\sum_{j=0}^{t} a^{j} f_{j}, f_{j} \in S$ and for every $k=1, \ldots, t$, let $g_{k}=\sum_{j=0}^{t} a_{k}^{j} f_{j}, f_{j} \in S$. Then, by Proposition P2, $\prod_{k=1}^{t} g_{k}$ belongs to $S$ and in the same way, $\prod_{k=2}^{t} g_{k}$ belongs to $\widehat{S}$. Now, since $\inf _{\mathbb{E}}|g(x)|$ is a number $m>0$, we have $\left|\prod_{k=1}^{t} g_{k}\right| \geq m^{t}$ because in $\mathbb{L}$, we have $\left|g_{k}(x)\right|=\left|g_{1}(x)\right| \forall k=1,2, \ldots, t, \forall x \in \mathbb{E}$. Consequently, $\prod_{k=1}^{t} g_{k}$ is invertible in $S$.

There exists $f \in S$ such that $\prod_{k=1}^{t} g_{k} . f=1$. Since by Proposition P2, $\prod_{k=2}^{t} g_{k}$ belongs to $\widehat{S}$ one sees that $\prod_{k=2}^{t} g_{k} . f$ is an element of $\widehat{S}$. Hence $g=g_{1}$ is invertible in $\widehat{S}$ with inverse $g^{-1}=\prod_{k=2}^{t} g_{k} \cdot f$

Definition and notation: In the following Proposition P4 and in the theorems we will have to consider the tensor product norm We remind here some general facts ( for completeness one can sees [9]). Let $\mathbb{L}$ be a complete valued field extension of $\mathbb{K}$ and $A$ be a unital, ultrametric $\mathbb{I K}$-Banach algebra. Given $z \in \mathbb{L} \otimes_{\mathbb{K}} A$, we put

$$
\|z\|_{\otimes}=\inf \left\{\max _{i \in I}\left|b_{i}\right| \cdot\left\|x_{i}\right\| \mid \sum_{i \in I} b_{i} \otimes_{\mathbb{K}} x_{i}=z, \text { Ifinite }\right\} .
$$

This norm $\|.\|_{\otimes}$ will be called the (projective) tensor product norm. It is an ultrametric norm. 
In any unital $\mathbb{K}$-algebra $A$, let $1_{A}$ be the unity of $A$. Then for $b \in \mathbb{L}$ and $x \in A$, one has $\|b \otimes x\|_{\otimes}=|b| \cdot\|x\|$. In particular for any $b \in \mathbb{L}$, (resp. $x \in A$ ), one has $\left\|b \otimes 1_{A}\right\|_{\otimes}=|b|$ (resp. $\left\|1_{\mathbb{K}} \otimes y\right\|_{\otimes}=\|x\|$.) Hence one has an isometric identification of $\mathbb{L}$ (resp. $A$ ) with $\mathbb{L} \otimes_{K} 1_{A}\left(\right.$ resp. $\left.1_{\mathbb{L}} \otimes_{\mathbb{K}} A\right)$.

Furthermore, one verifies that with the tensor norm, the tensor product $\mathbb{L} \otimes_{\mathbb{K}} A$, of the two unital $\mathbb{K}$-algebras $\mathbb{L}$ and $A$ is a normed unital $\mathbb{K}$-algebra. It is also a unital $\mathbb{L}$-algebra (obtained by extension of scalars). The completion $\mathbb{L} \widehat{\otimes}_{\mathbb{K}} A$ of $\mathbb{L} \otimes_{\mathbb{K}} A$ with respect to the tensor product norm $\|\cdot\|_{\otimes}$ (called the topological tensor product) is a unital $\mathbb{K}$-Banach algebra as well as a $\mathbb{L}$-Banach algebra.-

Now assume that $\mathbb{L}$ is of finite dimension $d$ over $\mathbb{K}$. Fix a $\mathbb{K}$-basis $\left(e_{j}\right)_{1 \leq j \leq d}$ of $\mathbb{L}$. It is readily seen that any $z \in \mathbb{L} \otimes_{\mathbb{K}} A$ can be written in the unique form $z=\sum_{j=1}^{d} e_{j} \otimes y_{j}$ and $\|z\|_{\otimes}=\left\|\sum_{j=1}^{d} e_{j} \otimes y_{j}\right\|_{\otimes} \leq \max _{1 \leq j \leq d}\left|e_{j}\right| \cdot\left\|y_{j}\right\|$.

On the other hand let us consider for $b=\sum_{j=1}^{d} \beta_{j} e_{j} \in \mathbb{L}$ the norm $\|b\|_{1}=\max _{1 \leq j \leq d}\left|\beta_{j}\right| \cdot\left|e_{j}\right|$. One has $|b| \leq\|b\|_{1}$ and since $\mathbb{L}$ is finite dimensional, there exists $\alpha>0$ such that $\alpha \max _{1 \leq j \leq d}\left|\beta_{j}\right| \cdot\left|e_{j}\right| \leq|b| \leq \max _{1 \leq j \leq d}\left|\beta_{j}\right| \cdot\left|e_{j}\right|$. Considering the dual basis $\left(e_{j}^{\prime}\right)_{1 \leq j \leq d}$ of $\left(e_{j}\right)_{1 \leq j \leq d}$ and the continuous linear operators $e_{j}^{\prime} \otimes i d_{A}$ of $\mathbb{L} \otimes_{\mathbb{K}} A$ into $\mathbb{K} \otimes_{\mathbb{K}} A=A$, one proves that $\alpha \max _{1 \leq j \leq d}\left|e_{j}\right| \cdot\left\|y_{j}\right\| \leq\|z\|_{\otimes} \leq \max _{1 \leq j \leq d}\left|e_{j}\right| \cdot\left\|y_{j}\right\|=\|z\|_{1}$.

That means that the norms $\|$. $\|_{\otimes}$ and $\|$. $\|_{1}$ of $\mathbb{L} \otimes_{\mathbb{K}} A$ are equivalent. One immediately sees that $\mathbb{L} \otimes_{\mathbb{K}} A$ equiped with the norm $\|z\|_{1}=\max _{1 \leq j \leq d}\left|e_{j}\right|\left\|y_{j}\right\|$ is isomorphic to the product $\mathbb{K}$-Banach space $A^{d}$ and then it is complete. It follows that $\left(\mathbb{L} \otimes_{\mathbb{K}} A, \|\right.$. $\left.\|_{\otimes}\right)$ is complete and $\mathbb{L} \otimes_{\mathbb{K}} A=\mathbb{L} \widehat{\otimes}_{\mathbb{K}} A$.

One then has the following Theorem B more or less contained in [9] (Chapter 4).

Theorem B: If $\mathbb{L}$ is a finite extension of $\mathbb{K}$ and $A$ is a commutative unital $\mathbb{K}$-Banach algebra, then with the tensor product norm $\|.\|_{\otimes}$, the tensor product $\mathbb{L} \otimes_{\mathbb{K}} A$ of the $\mathbb{K}$-algebras $\mathbb{L}$ and $A$ is a $\mathbb{K}$-Banach algebra as well as a Banach algebra over $\mathbb{L}$.

Taking $A=S$, we can now conclude.

Proposition P4 : Let $\mathbb{L}=\mathbb{K}[a]$ be a finite extension of $\mathbb{K}$ of degree $t$, provided with the unique absolute value extending that of $\mathbb{K}$. Then the algebra $\widehat{S}=\mathbb{L} \otimes_{\mathbb{K}} S$ provided with the tensor product norm \|\|$_{\otimes}$, is complete.

Moreover, $\widehat{S}$ can be identified with the Banach $\mathbb{L}$-algebra of functions $f$ from $\mathbb{E}$ to $\mathbb{L}$ of the form $f=\sum_{j=0}^{t-1} a^{j} f_{j}$ and $\widehat{S}$ is a semi-admissible (resp. semi-compatible) $\mathbb{L}$-algebra.

Proof: By construction, $\widehat{S}$ is the set of functions $f=\sum_{j=0}^{t-1} a^{j} f_{j}$ with $f_{j} \in S$. Since each 
$f_{j}$ is continuous (resp. uniformly continuous), so is $f$. By Theorem $\mathrm{B}, \widehat{S}$ is a Banach $\mathbb{L}$-algebra. Next, given a clopen subset set (resp. a uniformly open subset) $D$ of $\mathbb{E}$, the characteristic function $u$ of $D$ exists in $S$ and hence it belongs to $\widehat{S}$. Finally, given an element $g \in \widehat{S}$ such that $\inf _{x \in \mathbb{E}}|g(x)|>0$, by Proposition P3, $g$ is invertible in $\widehat{S}$. Therefore, $\widehat{S}$ is semi-admissible (resp. semi-compatible).

Theorem 1 : Let $\mathbb{L}$ be a finite extension of $\mathbb{K}$ of degree $t$, provided with the unique absolute value extending that of $\mathbb{K}$ and let $\widehat{S}=\mathbb{L} \otimes_{\mathbb{K}} S$ be provided with the tensor product norm. Then $\widehat{S}$ is a semi-admissible (resp. semi-compatible) Banach $\mathbb{L}$-algebra.

Proof: By definition, $\mathbb{L}$ is of the form $\mathbb{K}\left[b_{1}, \ldots, b_{q}\right]$ with $\mathbb{K}\left[b_{1}, \ldots, b_{j}\right]$ strictly included in $\mathbb{K}\left[b_{1}, \ldots, b_{j+1}\right], j=1, \ldots, q-1$. Put $\mathbb{L}_{j}=\mathbb{L}\left[b_{1}, \ldots, b_{j}\right], j=1, \ldots, q$ and $\widehat{S}_{j}=\mathbb{L}_{j} \otimes_{\mathbb{K}} S$. Suppose we have proved that $\widehat{S}_{j}$ is semi-admissible (resp. semi-compatible) for some $j<q$. Next, since $\mathbb{L}_{j+1}=\mathbb{L}_{j}\left[b_{j+1}\right]$, by Proposition P4, $\widehat{S}_{j+1}$ is semi-admissible (resp. semicompatible). Therefore, by induction, $\widehat{S}_{q}=\widehat{S}$ is a semi-admissible (resp. semi-compatible) Banach IL-algebra.

Theorem 2: $\quad$ Let $\mathcal{M}$ be a maximal ideal of finite codimension of $S$. Then $\mathcal{M}$ is of codimension 1 .

Proof: Let $\mathbb{L}$ be the field $\frac{S}{\mathcal{M}}$ and let $\widehat{S}=\mathbb{L} \otimes_{\mathbb{K}} S$ be provided with the tensor norm product. By Theorem 1, $\widehat{S}$ is semi-admissible (resp. semi-compatible). Now, let $\chi$ be the morphism from $S$ over $\mathbb{L}$ whose kernel is $\mathcal{M}$. Let $g \in S$ and let $b=\chi(g) \in \mathbb{L}$. By Proposition P1, $\chi$ admits an extension to a morphism $\widehat{\chi}$ from $\widehat{S}$ to $\mathbb{L}$. Now, since $\widehat{S}$ is semi-admissible (resp. semi-compatible) and since the kernel of $\widehat{\chi}$ is a maximal ideal $\widehat{\mathcal{M}}$ of $\widehat{S}$, by Proposition A there exists an ultrafilter $\mathcal{U}$ on $\mathbb{E}$ such that $\widehat{\mathcal{M}}=\mathcal{I}(\mathcal{U}, \widehat{S})$. Take $g \in S$ and let $b=\chi(g)$. Then we have $\widehat{\chi}(g-b)=0$, hence $g-b$ belongs to $\widehat{\mathcal{M}}$, therefore $\lim _{\mathcal{U}} g(x)-b=0$ i.e. $\lim _{\mathcal{U}} g(x)=b$. But since $g \in S, g(x)$ belongs to $\mathbb{K}$ for all $x \in \mathbb{E}$. Therefore, since $\mathbb{K}$ is complete, $b$ belongs to $\mathbb{K}$. But by definition $\chi$ is a surjection from $S$ onto $\mathbb{L}$, hence every value $b$ of $\mathbb{L}$ actually lies in $\mathbb{K}$ and hence $\mathbb{L}=\mathbb{K}$.

Let us now recall the following result that we can extract from Theorem 4.8 in [2]:

Theorem C: Let $\mathcal{M}$ be a maximal ideal of codimension 1 of $S$. Then the quotient norm of the field $\frac{S}{\mathcal{M}}$ is equal to the absolute value of $\mathbb{I K}$.

Theorem 3: $\quad$ Suppose $\mathbb{E} \subset \mathbb{K}$ and let $\mathcal{M}=\mathcal{I}(\mathcal{U}, S)$ be a maximal ideal of $S$ where $\mathcal{U}$ is an ultrafilter on $\mathbb{E}$ and suppose that the identical function belongs to $S$. If $\mathcal{U}$ is a Cauchy filter, then $\mathcal{M}$ is of codimension 1. Else, $\mathcal{M}$ is of infinite codimension.

Proof: Suppose first that $\mathcal{U}$ is a Cauchy ultrafilter. By Theorem $\mathrm{C}$, we can assume that $\mathcal{U}$ converges to a point $a$ of the completion $\widehat{\mathbb{E}}$ of $\mathbb{E}$ with respect to $\delta$. Then, by Theorem A, the ideal $\widehat{\mathcal{M}}$ is of codimension 1 and so is $\mathcal{M}$. Now, suppose that $\mathcal{U}$ is not a Cauchy filter. Consider the identical function $g$ defined on $\mathbb{E}$. Then $g$ has no limit on $\mathcal{U}$, therefore 
by Theorem A again, $\mathcal{M}$ is not of codimension 1 . But then by Theorem $2, \mathcal{M}$ is not of finite codimension.

Remark: In Proposition P2 and P3 we have to consider conjugates of an algebraic element over $\mathbb{I K}$. This is why we take a perfect field. It is an interesting conjecture that the theorems hold without that hypothesis.

\section{References:}

[1] Bourbaki, N., Topologie générale, Ch.3. Actualités scientifiques et industrielles, Hermann, Paris.

[2] Chicourrat, $\mathbf{M}$ and Escassut A., Banach algebras of ultrametric Lipschitzian functions, Sarajevo Journal of Mathematics, (2018).

[3] Escassut, A. The ultrametric Banach algebras. World Scientific Publishing Co (2003).

[4] Escassut, A. and Maïnetti, N. , Multiplicative spectrum of ultrametric Banach algebras of continuous functions Topology and its applications 157, p. 2505-25015 (2010).

[5] Escassut, A. and Maïnetti, N. , Morphisms between ultrametric Banach algebras and maximal ideals of finite codimension Contemporary Mathematics 596, p. 63-71 (2013)

[6] Escassut, A. and Maïnetti, N. , Spectrum of ultrametric Banach algebras of strictly differentiable functions Contemporary Mathematics 704, p. 139-160 (2018).

[7] Haddad, L. Sur quelques points de topologie générale. Théorie des nasses et des tramails. Annales de la Faculté des Sciences de Clermont N 44, fasc.7, p.3-80 (1972).

[8] Samuel P. , Ultrafilters and compactification of uniform spaces, Trans. Amer. Soc. 64, p. 100-132 (1949).

[9] Van Rooij, A. Non-Archimedean Functional Analysis. Marcel Dekker (1978).

Laboratoire de Mathématiques Blaise Pascal, UMR 6620

Université Clermont Auvergne

24 Avenue des Landais

3 Place Vasarely

63178 AUBIERE-CEDEX

FRANCE 